\title{
General Psychiatry Effectiveness of E-SBIRT intervention in community healthcare institution in China: study proposal for a randomised controlled trial
}

\author{
Rao Fu, ${ }^{1}$ Chenxin Yuan, ${ }^{1}$ Wei Sun, ${ }^{2}$ Wenzheng Wang, ${ }^{1}$ Lei Zhang, ${ }^{1}$ Jing Zhai, ${ }^{1}$ \\ Qun Guan, ${ }^{1}$ Xiaojun Wu, ${ }^{1}$ Jiang Long, ${ }^{3}$ Min Zhao (D) , Jiang Du ${ }^{1}$
}

To cite: Fu R, Yuan C, Sun W, et al. Effectiveness of E-SBIRT intervention in community healthcare institution in China: study proposal for a randomised controlled trial. General Psychiatry 2021;34:e100486. doi:10.1136/ gpsych-2021-100486

$\mathrm{RF}$ and $\mathrm{CY}$ contributed equally.

Received 03 March 2021 Accepted 20 August 2021

Check for updates

(c) Author(s) (or their employer(s)) 2021. Re-use permitted under CC BY-NC. No commercial re-use. See rights and permissions. Published by BMJ.

${ }^{1}$ Shanghai Mental Health Center, Shanghai Jiao Tong University School of Medicine, Shanghai, China

${ }^{2}$ Community Health Service Center of Jiangning Road, Shanghai, China

${ }^{3}$ Psychological Science Research Institute, Université Catholique de Louvain, Louvainla-Neuve, Belgium

Correspondence to Dr Jiang Du; dujiangdou@163.com

Dr Min Zhao; drminzhao@gmail.com

\section{ABSTRACT}

Background Many studies have demonstrated the effectiveness of Screening, Brief Intervention and Referral to Treatment (SBIRT) in addressing substance use problem. However, owing to the shortage of counsellors, it has not been widely used in China. With the development of smart medicine, we developed a web-based electronic SBIRT (ESBIRT) program and explored the effectiveness of E-SBIRT in reducing substance use in China.

Methods A randomised controlled trial will be conducted in primary healthcare institutions. Four primary healthcare institutions will be selected and randomly divided into an intervention group and a control group (each institution will recruit 60 participants, and in total, 240 participants will be recruited). The control group will get a pamphlet of drug abuse prevention, and the intervention group will get the E-SBIRT intervention and the pamphlet. Both groups will receive baseline and follow-up assessment at 1 and 3 months after the intervention. The primary outcome is the change in scores on the Alcohol, Smoking and Substance Use Involvement Screening Test, and the secondary outcomes include changes in motivation, depression, anxiety, positive/negative emotion, self-esteem, addiction knowledge and addiction severity index.

Conclusions If the 'E-SBIRT' program is found to be effective, it will be an accessible, affordable and widely implementable intervention to help participants at moderate risk of substance use to reduce their consumption. The potential benefit is to provide early intervention to high-risk patients in time and reduce the harmful consequences to individuals and society. Trial registration number NCT03452241.

\section{INTRODUCTION}

Substance use is one of the biggest risk factors for the global burden of disease, accounting for $11.5 \%$ of the total health burden. ${ }^{1}$ Addiction to illicit substances has become a serious public health problem, ${ }^{2}$ with significant social, health and economic consequences. ${ }^{3}$ Drug use around the world has been on the rise, in terms of both the overall number and the proportion of the world's population that uses drugs. In 2018, the estimated 269 million users represented $5.3 \%$ of the global population aged $15-64 .{ }^{4}$ By the end of 2019, there have been 2.14 million drug users nationwide, accounting for $0.16 \%$ of the total population in China; however, the actual number of drug users is much higher than this. ${ }^{5} \mathrm{~A}$ doseresponse relationship exists between alcohol use and many major diseases. ${ }^{6}$ More than 3 million people died in 2016 as a result of harmful use of alcohol, accounting for $5.3 \%$ of all deaths. Per capita alcohol consumption in China has risen and abstinence rate has fallen. In particular, the report notes that the number of cases of alcoholic liver disease in China is rising at an alarming rate. ${ }^{7}$ China accounts for more than $40 \%$ of the world's total cigarette consumption. ${ }^{8}$ The total number of smokers in China has increased compared with that at the end of the 20th century, and the age of first smoking has been declining. However, only $7.1 \%$ of those with substance use disorders received minimally adequate treatment. ${ }^{9}$ These substance abuse behaviours need to be screened, assessed and intervened to reduce the risks of substance addiction. However, there is still a lack of effective screening and intervention tools in China, which may lead to the delay of treatment. Community healthcare institutions in China have developed rapidly in recent years. By the end of June 2020, China had 35000 community healthcare institutions. ${ }^{10}$ Community healthcare institutions have the advantages of relatively fixed patients, convenient services, strong pertinence and other advantages, with "prevention of diseases, treatment of minor diseases, common diseases, major diseases referral' as the main responsibilities. ${ }^{11}$ Therefore, community healthcare institutions have become an advantageous platform for implementing screening and intervention technologies. Timely screening 
and early intervention in community are important measures to reduce the harm of substance abuse.

The Screening, Brief Intervention and Referral to Treatment (SBIRT) is a comprehensive approach proposed by the WHO to provide early intervention and treatment services through comprehensive screening of people with substance use disorders and those at risk. ${ }^{12}$ The SBIRT integrated intervention pattern consists of two steps. The first step is initial screening, and the Alcohol, Smoking and Substance Use Involvement Screening Test (ASSIST) is used as a screening tool. This process identifies people who are experiencing addiction-related risk or harm and provides personalised feedback on substance use and harms. The second step is to conduct a brief intervention or referral based on the screening results. Numerous studies have demonstrated the effectiveness of SBIRT aimed at addressing substance use problems and treatment needs among patients. ${ }^{13} 14$ China has also carried out relevant research. ${ }^{15}$ SBIRT is easy to operate, time consumable and efficient, and has been promoted in primary health institutions ${ }^{16}{ }^{17}$ and emergency departments. ${ }^{18}$ Unfortunately, surveys have found the utilisation of SBIRT to be low. ${ }^{19}$ There are some patients who are unwilling or unable to attend health service, ${ }^{20}$ and there are various other barriers that result in comparatively reduced rates of intervention, including poor or inadequate availability of services; concerns about privacy, embarrassment or dislike of answering personal questions; treatment expense or lack of insurance; and pragmatic issues, such as work-related complications and transportation problems. ${ }^{19}{ }^{21}$ It may also be owing to the heavy burden of healthcare that there are not enough resources to implement it. Therefore, a new way is needed to make up for the deficiency of traditional treatment.

With the popularisation of the internet and with technological progress, electronic-based intervention strategies have been widely used in the medical field and have proved to be successful in treating patients. ${ }^{22-24} \mathrm{~A}$ metaanalysis of electronic interventions for reducing harmful alcohol consumption suggests that electronic interventions may reduce alcohol consumption, with an average reduction of up to three (UK) standard drinks per week and that there may be little or no difference in impact on alcohol consumption between electronic and face-to-face interventions. ${ }^{25}$ Current programs are mostly designed for personal computers that are not readily accessible during a user's everyday routine. A study reported that a program designed for mobile devices was significantly more effective in encouraging smoking cessation than for non-mobile devices, and more participants in the mobile device group reported that they would recommend the program to their friends/families interested in quitting. ${ }^{26}$ Foreign scholars have developed an electronic SBIRT (E-SBIRT) and studied its effect. A study focused on substance-involved probationers suggested that motivational computer intervention, as part of SBIRT, can be used to address public safety and health issues. ${ }^{27}$ The studies of alcohol users and smokers demonstrated that
E-SBIRT promoted the initial contact with substance abuse treatment providers and encouraged those who referred to begin treatment, but it did not reduce risky substance use behaviour. ${ }^{28} 29$

To our knowledge, there is no E-SBIRT study in China. To fill this gap, we developed an E-SBIRT and aimed to test the effectiveness of this program in reducing substance abuse among community healthcare institutions. We assume that compared with the control group, ASSIST scores at 1-month and 3-month follow-up after baseline assessment will be significantly reduced in the 'E-SBIRT' intervention group.

\section{METHODS}

\section{Study design and participants}

The setting for this study is in Shanghai, China. There is a good foundation for community work. Four community healthcare institutions are randomly selected and are required to be basically comparable in terms of level of economic development, size, staffing and hardware configuration, geographical environment and demographic structure. Each institution is required to have more than 500 patients per month and has 60 participants enrolled. In order to reduce the impact of communication between the participants on the results and save the manpower needed for management, we use community randomisation. Four community hospitals are randomised via computerised assignment in a computer program to either the brief intervention group or the control group (1:1). Participants are blinded to random assignments. The participants in the intervention group will watch an intervention video, while the control group will not. All participants complete a baseline assessment before the intervention and follow-up assessments performed 1 and 3 months after the intervention.

\section{Participant eligibility criteria}

The inclusion criteria were (1) aged between 18 and 65 , with an ASSIST screening score of moderate risk (11-26 for alcohol, 4-6 for other substances); (2) voluntary participation and adept at using smartphones and WeChat; (3) able to access the internet; and (4) agree to cooperate with baseline and follow-up study. The exclusion criteria were severe cognitive impairment and inability to complete the questionnaire. Suspension criteria were (1) serious adverse events occurring during the test; (2) the ethics committee ceasing or suspending the approved experiments, having the right to do so; and (3) the investigator seriously violating the approved protocol or relevant regulations.

\section{Sample size calculation}

The sample size is calculated according to the primary outcome: variation in ASSIST scores at 3-month follow-up. Related literature pointed out that the use of SBIRT can result in a $16 \%$ reduction, and the control group has a 3\% reduction in ASSIST scores. Based on previous 
studies, the percentage of participants lost to follow-up is $20 \%$. Assuming $\alpha=0.05$ and $\beta=0.10$, a sample size of 240 participants is calculated by sample size estimation for a comparison of two groups.

\section{Recruitment}

Staff at community healthcare institutions invite outpatients to participate in the study. The first is the screening phase. When patients agree to participate in the study, they are invited to register for an account in E-SBIRT and provide basic demographic information. The patient then fills out the ASSIST scale, and upon completion, the patient's risk level for substance use (high, medium or low risk) is immediately displayed on the screen. Patients who scored between 11 and 26 on ASSIST (moderaterisk range) for alcohol and scored between 4 and 6 on ASSIST (moderate-risk range) for other substances are then approached by the research assistant for a written informed consent for enrolment in the intervention study. Participants who enrolled in this study could withdraw at any time. They will also be asked to provide a mobile phone number for follow-up assessments.

\section{Data collection}

In this trial, all outcome data collection (including baseline and each follow-up) will be carried out via E-SBIRT. After completing the questionnaire, the data will be automatically uploaded to the back-end server and stored with an identification number as the key index. Follow-up information will be collected in the same way.

\section{Baseline data}

Demographic information will be obtained from participants at baseline. The demographic information of participants will include gender, age, marital status, education level and working status.

\section{Alcohol, Smoking and Substance Involvement Screening Test}

The screening results are divided into three levels, with a score of 0-3 (0-10 for alcohol) as the low-risk group, indicating that the patient's current use of psychoactive substances is in a low-risk state; with a score of 4-6 (11-26 for alcohol) as the moderate-risk group, indicating that the patient's current use of psychoactive substances is at moderate risk; and with a score of $\geq 7$ ( $\geq 27$ for alcohol) as the high-risk group, indicating that the patient's current use of psychoactive substances is at a highrisk level, which may indicate that the patient's current psychoactive substance use raises serious health, social, economic, legal, interpersonal problems and is likely to be dependent. ${ }^{30}$

\section{Self-Rating Anxiety Scale}

The Self-Rating Anxiety Scale (SAS) is a self-report scale that consists of 20 items used to evaluate severity of anxiety. ${ }^{31}$ Each item is scored on a 4-point Likert-type scale ranging from 1 (a little of the time) to 4 (most of the time). A higher score indicates a higher level of anxiety.

\section{Self-Rating Depression Scale}

The Self-Rating Depression Scale (SDS) is a self-report scale that consists of 20 items used to evaluate severity of depression. ${ }^{32}$ Each item is scored on a 4-point Likerttype scale ranging from 1 (a little of the time) to 4 (most of the time). A higher score indicates a higher level of depression.

\section{Assessment Questionnaire on Addiction Knowledge}

We compiled a questionnaire on addiction knowledge by referring to relevant literature and intervention content. There are 23 questions in total, and the options are 'correct', 'wrong' and 'don't know'. The rate of accuracy for each participant is calculated.

\section{Rosenberg Self-Esteem Scale}

The Rosenberg Self-Esteem Scale (RSES) is a self-report scale that consists of 10 items used to evaluate the level of self-esteem. ${ }^{33}$ Each item is answered on a 4-point scale ranging from strongly agree to strongly disagree. The scale ranges from 0 to 30 . Scores below 15 suggest low self-esteem.

\section{Positive and Negative Affect Scale}

The Positive and Negative Affect Scale (PANAS) is a selfreport scale that consists of 20 items used to evaluate the mental health status of the community population and the psychological adjustment of clinical patients. ${ }^{34}$

\section{Pre-Treatment Readiness Scale}

The Pre-Treatment Readiness Scale (PRS) is a selfcomplied scale. The scale is based on a transtheoretical model of behavioural change developed by Prochaska and DiClemente. ${ }^{35}$ There are three dimensions: precontemplation, contemplation and action. Precontemplation is being completely unaware of the problem. Contemplation is when patients become aware of a problem and start thinking hard about how to overcome it, but do not act on it. Action is to make a change but the change does not last. Each dimension consists of four questions, scored on a 5-point scale, with 1 indicating 'strongly disagree' and 5 indicating 'strongly agree'. The higher the average score at a certain stage, the higher the probability that the respondents are at that stage.

\section{Addiction Severity Index}

The Addiction Severity Index (ASI) is a structured clinical interview developed to fill the need for a reliable, valid and standardised diagnostic and evaluative instrument in the field of alcohol and drug abuse. ${ }^{36}$ The study will use a few dimensions from the ASI as secondary efficacy indicators. The scale will be completed by the participants according to their own situation.

\section{Post-Study System Usability Questionnaire}

The Post-Study System Usability Questionnaire (PSSUQ) is used to evaluate users' perceived satisfaction with the computer system or application. It consists of 15 items, 


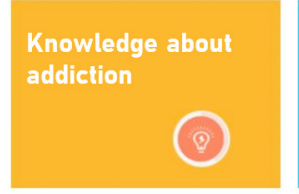

Screening
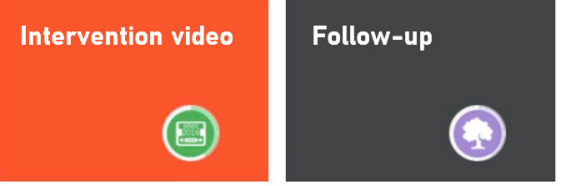

Figure 1 Main interface of the E-SBIRT program. E-SBIRT, electronic Screening,Brief Intervention and Referral to Treatment.

including an overall scale and 3 subscales of system quality, information quality and interface quality. ${ }^{37}$

\section{Development of the E-SBIRT program}

E-SBIRT is a web application that can be viewed using an embedded browser and accessed through WeChat. WeChat is the most popular multifunctional social media app in China, with 1.15 billion monthly active users or $80 \%$ of the Chinese population. ${ }^{38}$ It is easily accessible to users and does not require installation of additional software. E-SBIRT mainly includes four parts: knowledge about addiction, screening, intervention video and follow-up. The main interface of the program is shown in figure 1 .

There are three sections in 'knowledge about addiction': (1) what you need to know about addiction, (2) introduction of brief intervention technology, and (3) introduction of ASSIST. Users can choose whether or not to read this section according to their own interests.

The screening part consists of three steps. Users need to register an account the first time they use it. After logging in, it takes the user about $10 \mathrm{~min}$ to perform the ASSIST screening test. A score sheet and short speech feedback appear on the screen immediately after the test is completed. Feedback on test scores is categorised as low risk, moderate risk and high risk. If the user's score for all substances is in a low-risk state, it will indicate: 'You are currently in a low risk state for the use of addictive substances. It is recommended that you reduce the use of addictive substances and wish you good health!' If the user has a moderate-risk score, it indicates: 'You are currently at moderate risk for addictive substance use, and it is recommended that you receive the psychological intervention techniques provided by this software to help you reduce the harms associated with substance use'. If the user belongs to a high-risk group, it will indicate: 'You are currently at severe risk for addictive substance use, we suggest you go to the relevant medical institution for treatment!'

Participants at moderate risk watch a video on their mobile phones, which is about $15 \mathrm{~min}$ long. The video shows a researcher giving brief intervention to a patient at moderate risk of substance use. The brief intervention consists of nine steps (table 1).

The last part is a post-test to assess changes in the patient's condition.

\section{Intervention}

Participants who are allocated to the intervention group will watch the brief intervention video. After the intervention, participants will fill in the PRS and read the popular science pamphlet. Participants who are allocated to the control group will not watch the intervention video, but directly fill in the PRS and read the popular science pamphlet.

\section{Follow-up}

Both groups will finish follow-up assessments 1 and 3 months after the intervention. The questionnaires completed by the participants at baseline and follow-up are identical. Participants in the intervention group will take usability assessments at the 3-month follow-up. At the

\section{Table 1 Nine steps for brief interventions}

\begin{tabular}{|c|c|c|}
\hline Step 1 & Feedback on assessment results & $\begin{array}{l}\text { Give feedback on the results of ASSIST to the patients and make patients } \\
\text { aware of the risks of substance use. }\end{array}$ \\
\hline Step 2 & Recommendations & $\begin{array}{l}\text { Let patients establish a connection between reducing substance use and } \\
\text { reducing injury. }\end{array}$ \\
\hline Step 4 & Pay attention to the score on ASSIST & Encourage patients to express their concerns about substance abuse. \\
\hline Step 5 & Benefits of substance use & $\begin{array}{l}\text { Let patients know the benefits of substance use and discuss the positive } \\
\text { aspects of substance use. }\end{array}$ \\
\hline Step 7 & Summary & $\begin{array}{l}\text { Consolidate brief intervention, present difference and make the patient } \\
\text { produce cognitive conflict. }\end{array}$ \\
\hline Step 8 & $\begin{array}{l}\text { Focus on the negative consequences of } \\
\text { substance abuse }\end{array}$ & $\begin{array}{l}\text { Make the patient aware of the current or subsequent problems caused by } \\
\text { substance abuse. }\end{array}$ \\
\hline
\end{tabular}

ASSIST, Alcohol, Smoking and Substance Use Involvement Screening Test. 


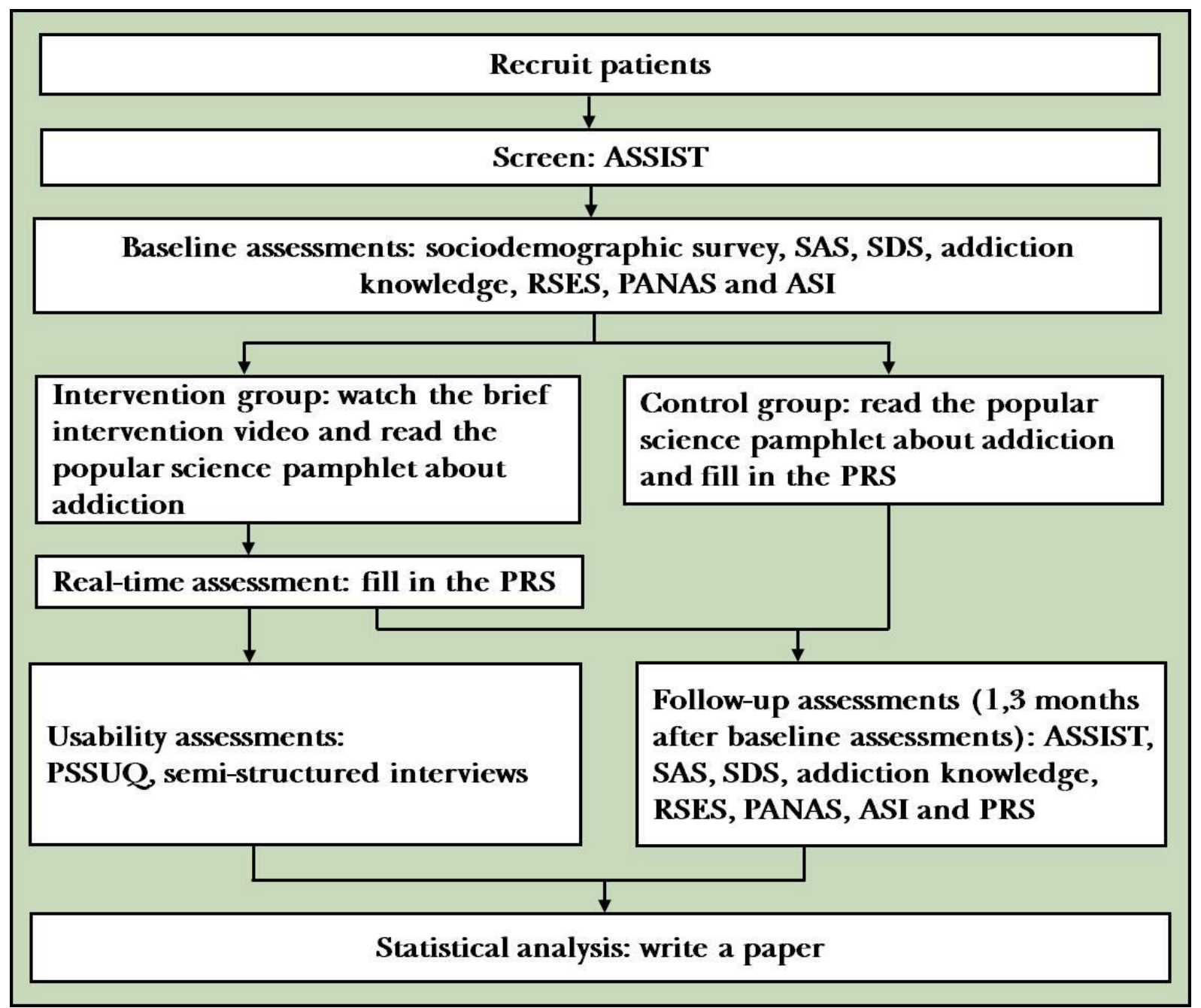

Figure 2 Flowchart of the study. ASI, Addiction Severity Index; ASSIST, Alcohol, Smoking and Substance Use Involvement Screening Test; PANAS, Positive and Negative Affect Scale; PRS, Pre-Treatment Readiness Scale; PSSUQ, Post-Study System Usability Questionnaire; RSES, Rosenberg Self-Esteem Scale; SAS, Self-Rating Anxiety Scale; SDS, Self-Rating Depression Scale.

same time, semi-structured interviews will be conducted. Patients who do not respond will receive phone calls as a reminder.

The procedure of the study is shown in a flowchart in figure 2 .

\section{Outcome measures}

The primary outcome is the change in ASSIST scores from baseline to 3-month follow-up.

The secondary outcome measures will be knowledge enhancement of addiction and changes in SAS, SDS, RSES, PANAS, ASI and PRS scores at 1-month and 3-month follow-up time points.

\section{Withdrawal from the program}

Patients have the right to withdraw from the study at any time and for any reason. Statistical analysis will be performed on an intention-to-treat basis. Participants who indicate that they are unwilling to participate in the study anymore by requesting to withdraw will be excluded from the analyses.

\section{Statistical analyses}

All data will be automatically collected by a data acquisition system into a database. All data will be stored in a password-protected network server with daily back-up. Data analysis is done using the Statistical Package for Social Sciences 23 (SPSS 23). The significant level will be set at $\mathrm{p}<0.05$ (two-tailed) for statistical hypothesis. The primary outcome analysis was based on the intention to treat. We will address missing outcomes by multiple imputation models. Descriptive analyses will be conducted to determine whether the randomisation procedure resulted in two groups having no differences in the distribution of demographic factors. Multiple comparison corrections will be conducted if necessary. In order to assess the initial efficacy of the program, the intervention group and control group will be compared on baseline characteristics to assess randomisation, and variables for which there are significant group differences will be controlled in subsequent analyses. $\chi^{2}$ test, repeated measure analysis of variance with unequal time interval and simple effect 
analysis were used for the analyses of baseline, 1-month and 3-month follow-up data.

Referring to other electronic intervention studies, ${ }^{39}$ we may face substantial attrition at follow-up because follow-up is more difficult when the intervention is received remotely without any interaction with trial personnel. ${ }^{25}$ To reduce attrition rates, medical staff will call participants to remind them to complete follow-up assessments. If the withdrawal rate is too high to meet the requirements of this study, we will report to the institutional review board and recruit participants according to the protocol requirements.

\section{Safety and adverse events}

Adverse events and serious adverse events will be recorded and documented. Participants can communicate with us via email and telephone in case they have questions or adverse events. If participants experience severe physical or mental withdrawal symptoms, they will be encouraged to see a healthcare provider.

\section{STRENGTHS AND LIMITATIONS OF THIS STUDY}

To our knowledge, this will be the first randomised controlled trial to evaluate the efficacy of E-SBIRT in China. However, there are some caveats to be considered. First, in studies that lack face-to-face contact, attrition rates are usually high. Thus, this study will reduce attrition rate by telephone reminders from staff. In addition, participants will be given a small gift for each baseline or follow-up assessment. Second, participants (especially those who are allocated to the control group) are allowed to access other intervention services. Finally, self-reported substance use is not validated by biochemical measures. However, biochemical validation is impractical in this study. We use multiple self-reported measures to make estimates of substance use more accurate.

\section{Twitter Jiang Long @Jiang_Long}

Acknowledgements We gratefully acknowledge the study participants who took their valuable time to participate in this study.

Contributors JD, MZ, RF and CY conceived and designed the protocol and will lead the study implementation. QG, LZ, XW, WS, JZ, JL and WW revised the study and assessment methods. All authors revised the protocol critically for important intellectual content.

Funding This work was supported by the Shanghai Mental Health Center (SMHC) Clinical Research Center Project (CRC2017YB04).

Competing interests None declared.

Patient consent for publication Not required.

Ethics approval The intervention will be carried out according to bioethical guidelines; the participants need to answer the questionnaires themselves and confidentiality is guaranteed. Ethics approval was received from the Institutional Review Board of Shanghai Mental Health Center (SMHC-IRB; number 2018-59). Prior to screening, all participants will have electronic informed consent.

Provenance and peer review Not commissioned; externally peer reviewed.

Data availability statement № data are available.

Open access This is an open access article distributed in accordance with the Creative Commons Attribution Non Commercial (CC BY-NC 4.0) license, which permits others to distribute, remix, adapt, build upon this work non-commercially, and license their derivative works on different terms, provided the original work is properly cited, appropriate credit is given, any changes made indicated, and the use is non-commercial. See: http://creativecommons.org/licenses/by-nc/4.0/.

ORCID iD

Min Zhao http://orcid.org/0000-0001-8382-3050

\section{REFERENCES}

1 GBD 2015 Risk Factors Collaborators. Global, regional, and national comparative risk assessment of 79 behavioural, environmental and occupational, and metabolic risks or clusters of risks, 1990-2015: a systematic analysis for the global burden of disease study 2015 . Lancet 2016;388:1659-724.

2 World Health Organization. Atlas on substance use (2010): resources for the prevention and treatment of substance use disorders. Geneva: World Health Organization, 2010.

3 Falcón M, Navarro-Zaragoza J, García-Rodríguez RM, et al. Perceived barriers to implementing screening and brief intervention for alcohol consumption by adolescents in hospital emergency department in Spain. Adicciones 2018;30:189-96.

4 UNODC world drug report, 2020. Available: https://wdr.unodc.org/ wdr2020/

5 Drug situation in China, 2019. Available: http://www.gov.cn/xinwen/ 2020-06/28/content_5522443.htm

6 GBD 2016 Alcohol and Drug Use Collaborators. The global burden of disease attributable to alcohol and drug use in 195 countries and territories, 1990-2016: a systematic analysis for the global burden of disease study 2016. Lancet Psychiatry 2018;5:987-1012.

7 World Health Organization. Global status report on alcohol and health 2018. Available: https://www.who.int/substance_abuse/publications/ global_alcohol_report/gsr_2018/en/

8 Yang G, Wang Y, Wu Y, et al. The road to effective tobacco control in China. Lancet 2015;385:1019-28.

9 Degenhardt L, Glantz M, Evans-Lacko S, et al. Estimating treatment coverage for people with substance use disorders: an analysis of data from the world mental health surveys. World Psychiatry 2017;16:299-307.

10 National Health Commission of China. China health statistical Yearbook. National Health Commission of China, 2019.

11 Zhong-can W, Xuelian C, Zhongchen H. Discussion on healthy cultural construction pathway based on healthy Chinese strategy. Chin J Health Educ 2018;34:189-91.

12 Babor TF, McRee BG, Kassebaum PA, et al. Screening, brief intervention, and referral to treatment (SBIRT). Subst Abus 2007;28:7-30.

13 Rollnick S, Miller WR. What is motivational interviewing? Behav Cogn Psychother 1995;23:325-34.

14 Aldridge A, Linford R, Bray J. Substance use outcomes of patients served by a large US implementation of screening, brief intervention and referral to treatment (SBIRT). Addiction 2017;112(Suppl 2):43-53.

15 Wang D. Hazardous and harmful drinking among adults in Liuyang rural community: epidemiological survey and intervention trial. Zhongnan University, 2013.

16 Madras BK, Compton WM, Avula D, et al. Screening, brief interventions, referral to treatment (SBIRT) for illicit drug and alcohol use at multiple healthcare sites: comparison at intake and 6 months later. Drug Alcohol Depend 2009;99:280-95.

17 Karno MP, Rawson R, Rogers B, et al. Effect of screening, brief intervention and referral to treatment for unhealthy alcohol and other drug use in mental health treatment settings: a randomized controlled trial. Addiction 2021:116:159-69.

18 Bruguera P, Barrio P, Oliveras C, et al. Effectiveness of a specialized brief intervention for at-risk drinkers in an emergency department: short-term results of a randomized controlled trial. Acad Emerg Med 2018;25:517-25.

19 Cohen E, Feinn R, Arias A, et al. Alcohol treatment utilization: findings from the National epidemiologic survey on alcohol and related conditions. Drug Alcohol Depend 2007;86:214-21.

20 Brown J, West R, Angus C, et al. Comparison of brief interventions in primary care on smoking and excessive alcohol consumption: a population survey in England. Br J Gen Pract 2016;66:e1-9.

21 Grant BF. Barriers to alcoholism treatment: reasons for not seeking treatment in a general population sample. J Stud Alcohol 1997;58:365-71.

22 Riper $\mathrm{H}$, Hoogendoorn A, Cuijpers P, et al. Effectiveness and treatment moderators of internet interventions for adult problem drinking: an individual patient data meta-analysis of 19 randomised controlled trials. PLoS Med 2018;15:e1002714. 
23 Lau Y, Klainin-Yobas P, Htun TP, et al. Electronic-based lifestyle interventions in overweight or obese perinatal women: a systematic review and meta-analysis. Obes Rev 2017;18:1071-87.

24 van Luenen S, Garnefski N, Spinhoven P, et al. Guided internetbased intervention for people with HIV and depressive symptoms: a randomised controlled trial in the Netherlands. Lancet HIV 2018;5:e488-97.

25 Kaner EF, Beyer FR, Garnett C, et al. Personalised digital interventions for reducing hazardous and harmful alcohol consumption in community-dwelling populations. Cochrane Database Syst Rev 2017;9:CD011479.

26 Danaher BG, Tyler MS, Crowley RC, et al. Outcomes and device usage for fully automated Internet interventions designed for a smartphone or personal computer: the MobileQuit smoking cessation randomized controlled trial. J Med Internet Res 2019;21:e13290.

27 Lerch J, Walters ST, Tang L, et al. Effectiveness of a computerized motivational intervention on treatment initiation and substance use: results from a randomized trial. J Subst Abuse Treat 2017;80: :59-66.

28 Haskins BL, Davis-Martin R, Abar B, et al. Health evaluation and referral assistant: a randomized controlled trial of a web-based screening, brief intervention, and referral to treatment system to reduce risky alcohol use among emergency department patients. $J$ Med Internet Res 2017;19:e119.

29 Boudreaux ED, Abar B, Haskins B, et al. Health evaluation and referral assistant: a randomized controlled trial to improve smoking cessation among emergency department patients. Addict Sci Clin Pract 2015;10: :24.
30 WHO ASSIST Working Group. The alcohol, smoking and substance involvement screening test (ASSIST): development, reliability and feasibility. Addiction 2002;97:1183-94.

31 Zung WW, William WKZMD. A rating instrument for anxiety disorders. Psychosomatics 1971;12:371-9.

32 Zung WW. A self-rating depression scale. Arch Gen Psychiatry 1965;12:63-70.

33 Rosenberg M. Society and the Adolescent Self-Image. 1 edn. Princeton University Press, 1965

34 Watson D, Clark LA, Tellegen A. Development and validation of brief measures of positive and negative affect: the PANAS scales. $J$ Pers Soc Psychol 1988;54:1063-70.

35 Prochaska JO, DiClemente CC. Stages and processes of self-change of smoking: toward an integrative model of change. J Consult Clin Psychol 1983;51:390-5.

36 McLellan AT, Luborsky L, Woody GE, et al. An improved diagnostic evaluation instrument for substance abuse patients. The addiction severity index. J Nerv Ment Dis 1980;168:26-33.

37 Lewis JR. Psychometric evaluation of the Post-Study system usability questionnaire: the PSSUQ. Proceedings of the Human Factors Society Annual Meeting 1992;36:1259-60.

38 WeChat active in job creation in 2019: report, 2020. Available: https:// global.chinadaily.com.cn/a/202005/17/WS5ec10a05a310a8b241 156412.html [Accessed 17 May 2020].

39 Taylor GMJ, Dalili MN, Semwal M, et al. Internet-based interventions for smoking cessation. Cochrane Database Syst Rev 2017;9:Cd007078.

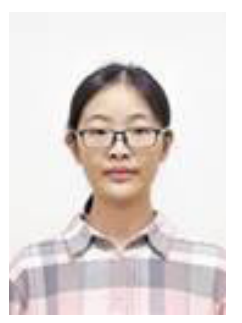

Rao Fu obtained a bachelor's degree from Jilin University in China in 2019. In the same year, she began her postgraduate studies in the Department of Substance and Addiction at the Shanghai Mental Health Center, Shanghai Jiao Tong University School of Medicine, China. She majors in social psychology, and her main research interest includes substance and addiction. 